\title{
Um estudo sobre desempenho escolar e motivação de crianças
}

\section{Academic performance and motivation of children study}

\author{
Selma de Cássia Martinelli ${ }^{1}$
}

\begin{abstract}
RESUMO
A motivação tem permeado as discussões dos profissionais da educação uma vez que à sua ausência tem sido atribuída uma queda de investimento pessoal do aluno na realização das tarefas de aprendizagem. Assim, o presente estudo teve por objetivo avaliar a motivação e o desempenho em leitura, escrita e aritmética de estudantes e investigar a relação entre as variáveis. Participaram da pesquisa 127 estudantes de uma escola pública do interior de São Paulo, de ambos os sexos, com idades entre 7 e 12 anos e que cursavam o $3^{\circ}, 4^{\circ}$ e $5^{\circ}$ ano escolar. Foram utilizados dois instrumentos de avaliação; a Escala de Avaliação da Motivação Escolar (EAME-IJ) e o Teste de Desempenho Escolar (TDE). Os resultados revelaram relações entre o desempenho em escrita e a motivação intrínseca e extrínseca dos estudantes. O desempenho escolar geral também se correlacionou com a motivação intrínseca e a motivação extrínseca dos estudantes.

Palavras-chave: variáveis educacionais; rendimento escolar; aprendizagem; ensino fundamental.
\end{abstract}

\begin{abstract}
Motivation has permeated the discussions of education professionals since its absence has been attributed to a drop in the student's personal investment in the tasks of learning. Thus, the present study was aimed to assess the motivation and performance in reading, writing and arithmetic of students and to investigate the relationship between the variables. 127 students of a public school in upstate São Paulo, of both sexes, aged 7-12 years and
\end{abstract}

DOI: $10.1590 / 0104-4060.27122$

1 Universidade Estadual de Campinas. Campinas. São Paulo, Brasil. Departamento de Psicologia Educacional da Faculdade de Educação. Rua Bertrand Russell, 801. CEP: 13083-865. 
attending the $3 \mathrm{rd}$, 4th and 5th school grade participated. Two instruments were used for evaluation; a Rating Scale of School Motivation (EAME-IJ) and an Academic Achievement Test (TDE). The results revealed the relationship between writing performance and intrinsic and extrinsic motivation of students and the overall school performance also correlated with intrinsic motivation and extrinsic motivation of students.

Keywords: educational variables; academic achievement; learning; elementary schooling.

\section{Introdução}

Nas últimas décadas verifica-se uma retomada dos estudos sobre motivação e sobre esta no contexto escolar. Grande parte das contribuições é decorrente da teoria da autodeterminação, proposta por Deci e Ryan (1985). Este modelo teórico tem centrado seus estudos nas condições sociocontextuais que facilitam ou enfraquecem os processos naturais de automotivação e de desenvolvimento saudável. Nessa perspectiva teórica três necessidades psicológicas básicas são consideradas fundamentais para promover esses processos naturais de motivação; a necessidade de autonomia, competência e de pertencimento ou estabelecimento de vínculos que, quando satisfeitas, promovem maior motivação e saúde mental. Assim o estudo destas necessidades tem sido de interesse de diversas áreas do conhecimento como a da saúde, trabalho, psicoterapia, educação, entre outras (RYAN; DECI, 2000).

Assim, pode-se dizer que, apesar de o ser humano apresentar uma disposição fenotípica para ser agente, ou seja, ter uma disposição para aprender, dominar novas habilidades, ampliar seus talentos, entre outras possibilidades, também é evidente que as pessoas diferem entre si nessas características, o que levou este modelo teórico a considerar que essas diferenças não são apenas decorrentes de dotes biológicos, mas que indicam que podem ser influenciadas por uma gama de variáveis contextuais. Dessa forma esta teoria tem se detido a especificar quais seriam as condições que favorecem estas atividades naturais e quais promoveriam essa falta de motivação e integração social. Nesse modelo teórico a motivação é vista como um componente energético do funcionamento individual e que atua na direção, persistência e busca da finalidade, considerados aspectos da ativação e intenção (RYAN; DECI, 2000).

De acordo com a Teoria da Autodeterminação as pessoas são motivadas por duas principais orientações; a extrínseca e a intrínseca. A orientação intrín- 
seca refere-se à inclinação natural para a assimilação, o domínio, o interesse e a exploração espontâneos (RYAN; DECI, 2000), enquanto a motivação extrínseca tem sido identificada como a motivação para trabalhar em resposta a algo externo à tarefa, assim como para a obtenção de recompensas materiais ou sociais. Os autores ainda consideram que, embora a motivação intrínseca seja um tipo importante de motivação, ela não é o único tipo de motivação autodeterminada e que, à medida que crescemos grande parte das coisas que fazemos não são motivadas estritamente de forma intrínseca.

Ainda de acordo com esta teoria há diferentes manifestações de motivação extrínseca, e elas refletem diferentes graus de internalização e de influência sobre o comportamento. Nesse sentido Ryan, Connell e Deci (1985) criticam a dicotomia utilizada nas definições de motivação intrínseca e extrínseca. Tais autores não questionam as características de autonomia e/ou de autorregulação, essenciais para a determinação da motivação intrínseca, mas acreditam que não é adequado afirmar que o comportamento extrinsecamente motivado não possa ser autodeterminado. Assim a questão principal que se coloca é sobre como as práticas motivacionais não intrínsecas podem afetar a persistência, a qualidade comportamental e o bem-estar.

Por ser atribuído à motivação um papel central no funcionamento individual, por sua característica de produção, é que profissionais de diversas áreas têm se ocupado dela. Esse mesmo interesse também tem estado presente nos profissionais da educação por se constatar que a motivação interfere consideravelmente no desempenho escolar dos alunos (BZUNECK, 2001). Essa mesma constatação pode ter levado Sá (2004) a afirmar que um estudante motivado considera a atividade escolar como parte significante e importante do seu projeto de vida e atribui um significado pessoal e positivo à própria aprendizagem, faz avaliações contínuas se é bom ou mau aluno, se tem um jeito especial para uma determinada tarefa e avalia a importância que estes resultados têm para si e para os outros. Da mesma maneira Guimarães e Boruchovitch (2004) afirmaram que um estudante motivado mostra-se ativamente envolvido no processo de aprendizagem, engaja-se e persiste em tarefas desafiadoras, usa estratégias adequadas e busca desenvolver novas habilidades de compreensão e de domínio. Apresenta ainda entusiasmo na execução das tarefas e orgulho acerca dos resultados de seu desempenho.

Embora os estudos sobre a motivação tenham apontado que as duas orientações motivacionais estejam presentes nos indivíduos e que ambas têm um papel importante no engajamento dos mesmos, tanto nas atividades corriqueiras quanto nas mais elaboradas, tem-se advogado mais a favor da necessidade de incrementar a motivação intrínseca e por conseguinte formas mais autônomas e autorreguladas de comportamento. Esse argumento tem sido justificado de 
diversas formas e também tem sido aplicado ao contexto escolar por se considerar que alunos intrinsecamente motivados optam mais por atividades que aprimoram suas habilidades, buscam novas informações, empenham-se em organizar o novo conhecimento, de acordo com seu conhecimento prévio, e procuram aplicá-lo em outros contextos, enquanto os alunos extrinsecamente motivados acreditam que o envolvimento na tarefa trará benefícios como, por exemplo, elogios ou prêmios.

Nesta mesma direção Ryan, Connell e Grolnick (1992) afirmaram que um bom desempenho escolar está associado a formas de autorregulação mais autônomas e à motivação intrínseca, ou seja, interiorização dos valores associados ao trabalho escolar. Por outro lado, estudantes com baixo desempenho escolar apresentam formas de regulação menos autônomas. Formas mais autodeterminadas de regulação ocorrerão se a criança compreender o valor da atividade, se lhes forem permitidas escolhas na realização das atividades com o mínimo de pressão, e se seus sentimentos e perspectivas forem reconhecidos. A valorização das tarefas resulta da interiorização e da integração, o que requer que os estudantes sintam-se competentes e percebam-se como autônomos durante a realização das atividades.

Apesar dessas controvérsias os dois padrões motivacionais vêm sendo investigados ao longo dos anos, por se compreender que essa variável apresenta-se como favorável à aprendizagem. Também não se sabe como o contexto escolar contribui para as mudanças ocorridas nas orientações motivacionais dos estudantes, embora venha se verificando um progressivo e significativo declínio na motivação intrínseca dos estudantes no decorrer do ensino fundamental e médio. Esses resultados são corroborados, entre outros, por estudos como o realizado por Lepper, Corpus e Iyengar (2005), que tiveram como um dos objetivos examinar a relação entre motivação intrínseca e extrínseca através de medidas independentes desses dois constructos no decorrer da escolarização. Os autores encontraram que a motivação intrínseca realmente decresceu, do $3^{\circ}$ para o $8^{\circ}$ grau, nas escolas americanas. $O$ mesmo resultado foi encontrado por Otis, Grouzet e Pelletier (2005), que se propuseram a investigar as mudanças nas orientações motivacionais de estudantes. Neste estudo constatou-se que houve um decréscimo gradual da motivação intrínseca e extrínseca dos alunos, do $8^{\circ}$ para o $10^{\circ}$ grau.

Mais recentemente, Corpus, McClintic-Gilbert e Hayenga (2009) investigaram a natureza e as mudanças na motivação durante um ano escolar, em uma amostra de estudantes do terceiro até o oitavo ano. As análises revelaram que houve mudanças nas orientações motivacionais intrínsecas e extrínsecas dos alunos durante o ano escolar. A diminuição da motivação intrínseca foi mais pronunciada nos adolescentes e declínios na motivação extrínseca ocorreram, 
de forma mais pronunciada, para os alunos do ensino fundamental. A motivação intrínseca e as atividades na sala de aula pareceram influenciar uma à outra de uma forma positiva e recíproca. Desempenhos em sala de aula mais pobres predisseram minimamente níveis mais altos de motivação extrínseca, embora esta não tenha sido apontada como a fonte de notas mais baixas.

O principal objetivo de Gillet, Vallerand e Lafrenière (2012) foi investigar a motivação intrínseca e extrínseca na escola e a ausência de motivação, em uma amostra de estudantes do ensino fundamental e médio, com idades entre 9-17 anos. Os resultados revelaram uma diminuição sistemática da motivação intrínseca e da motivação extrínseca autodeterminada, dos 9 aos 12 anos; uma estabilização lenta até 15 anos de idade, seguida por um aumento após esse ponto. Em segundo lugar, a motivação extrínseca, não autodeterminada, diminuiu até os 12 anos de idade e estabilizou-se após esse ponto. Finalmente, a motivação foi relativamente baixa e estável desde a idade de 9 até 17 anos.

Investigações sobre a motivação e o aproveitamento do aluno diante das aprendizagens escolares também tem sido conduzidas há várias décadas, em diversos países. Estes estudos têm apontado para a relevância da motivação para o desempenho e aprendizagem de estudantes, como verificado no trabalho conduzido por Gottfried et al. (2007), que investigaram, num estudo longitudinal, a motivação intrínseca para a matemática acadêmica e o desempenho em matemática de estudantes, com idades entre 9-17 anos. Em média, tanto a motivação quanto o desempenho em matemática diminuíram ao longo do tempo, desde a infância até a adolescência.

Estudos na perspectiva da teoria da autodeterminação, e que se propuseram a verificar como a motivação se coloca no decorrer do processo de escolarização, também foram conduzidos, no Brasil, por Neves e Boruchovitch (2004), Higa e Martinelli (2006), Martinelli (2008), Martinelli e Sisto (2010), e com exceção do primeiro estudo, os demais revelaram um certo declínio da motivação com o avanço da escolaridade, confirmando alguns achados da literatura internacional. Da mesma maneira estudos têm sido conduzidos com o intuito de avaliar as relações entre motivação e desempenho. Esse objetivo foi verificado no trabalho de Costa (2005) com estudantes com idades entre 11 e 12 anos, alunos do $8^{\circ}$ ano, em relação à disciplina de matemática. Os resultados indicaram que os estudantes apresentaram uma tendência à orientação motivacional predominantemente intrínseca. Também apontaram que os estudantes destacaram o valor do estudo e os benefícios deste como valor em si mesmo, ressaltando o esforço, a novidade e a curiosidade como importantes para a aprendizagem. Os dados indicaram ainda que alunos que estudavam somente para aprender apresentaram melhor desempenho na prova de matemática em relação aos outros alunos. 
As relações entre orientações motivacionais e o desempenho escolar, avaliado por meio de uma prova proposta pelo Sistema de Avaliação de Rendimento Escolar do Estado de São Paulo (SARESP), também foram investigadas por Martinelli e Genari (2009). Participaram do estudo estudantes que cursavam o $4^{\circ}$ e o $5^{\circ}$ ano do ensino fundamental. Os resultados revelaram que, no $4^{\circ}$ ano, as duas variáveis caminharam de forma independente. Já, no $5^{\circ}$ ano, os resultados evidenciaram que quanto maior a motivação intrínseca melhor o desempenho acadêmico apresentado pelos alunos. Os resultados indicaram ainda que, tanto no $4^{\circ}$ como no $5^{\circ}$ ano, quanto maior a motivação extrínseca menor o desempenho acadêmico apresentado pelos alunos.

Paiva e Boruchovitch (2010) também se propuseram a investigar, entre outras variáveis, as orientações motivacionais e o desempenho acadêmico de estudantes do quarto e do sexto ano do ensino fundamental. Quanto à motivação, as autoras identificaram que a maioria dos alunos que participou do estudo apresentou uma orientação motivacional predominantemente intrínseca $(77,5 \%)$, embora tenham observado um declínio da mesma ao longo dos anos. Também apontam para a prevalência da motivação intrínseca em estudantes com melhor desempenho.

Em síntese, pode-se inferir, a partir dessas pesquisas empíricas, que a motivação tem se mostrado correlacionada ao desempenho acadêmico de estudantes, o que revela a importância de se investigar essa variável em outros estudos, com outras amostras e outras medidas de desempenho. Também se verifica que poucos estudos foram conduzidos com o intuito de avaliar as relações entre o desempenho em diferentes áreas do conhecimento escolar e as orientações motivacionais dos alunos e, conforme apontado por Gottfried (1985), seria interessante que a motivação fosse estudada de forma específica para cada área do conhecimento ou conteúdos escolares específicos. Nesse sentido é que esta investigação se justifica, uma vez que se propôs a investigar o desempenho em leitura, escrita e aritmética, aferido pelo Teste de Desempenho Escolar (TDE), e suas relações com a motivação intrínseca e extrínseca.

Conforme apontado anteriormente, no presente estudo, não se trabalhou na perspectiva de dicotomia entre as orientações motivacionais intrínsecas e extrínsecas e sim com a ideia de que as duas orientações motivacionais possam atuar, concomitantemente, no funcionamento individual. No entanto, considerando os argumentos de que a motivação intrínseca apresenta-se como mais favorável e relacionada positivamente a um melhor desempenho escolar dos estudantes, este estudo se propôs a investigar como se relacionam essas duas orientações motivacionais com o desempenho escolar de crianças em áreas específicas do conhecimento. 


\section{Método}

\section{Participantes}

Foram avaliados 127 estudantes, sendo 57 (44,9\%) do sexo masculino e 70 $(55,1 \%)$ do sexo feminino, na faixa etária entre 7 e 12 anos (média de 8,95 anos), alunos do $3^{\circ}$ ao $5^{\circ}$ anos do ensino fundamental, de uma escola da rede pública de um município do interior do Estado de São Paulo. Compuseram a amostra estudantes de 10 salas de aula do $3^{\circ}, 4^{\circ}$ e $5^{\circ}$ anos. Nem todos os participantes do estudo realizaram na sua integralidade os testes propostos, o que resulta em números de participantes diferentes para cada instrumento.

\section{Instrumentos}

Teste de Desempenho Escolar - TDE (STEIN, 1994). O instrumento é composto pelos subtestes de leitura, escrita e aritmética e oferece uma avaliação no que diz respeito aos conteúdos e à série cursada pelo estudante. No subteste de escrita, a criança é solicitada a escrever o seu nome próprio e algumas palavras isoladas, apresentadas sob forma de ditado. O subteste de aritmética visa à solução oral de problemas matemáticos e à solução por escrito de cálculos de operações aritméticas e o subteste de leitura avalia a capacidade da criança em decodificar palavras isoladas, independente de seu significado. O Escore Bruto de acertos no subteste de leitura, escrita, aritmética e no geral é respectivamente de $70,35,38$ e 143 pontos.

Escala de avaliação da motivação escolar infanto-juvenil - EAME-IJ (MARTINELLI; SISTO, 2011). O Instrumento contém 20 itens que avaliam a motivação intrínseca e extrínseca dos estudantes, sendo 10 itens referentes a cada uma das orientações motivacionais. Cada item apresentava 3 possibilidades de resposta: "sempre" ( 2 pontos), "às vezes" ( 1 ponto) ou "nunca" ( 0 ponto), indicativas da frequência com que o estudante se autodeclarava motivado para realizar as tarefas escolares, podendo totalizar 20 pontos para cada uma das escalas.

Exemplos de itens da escala de motivação intrínseca

Quanto mais difícil o trabalho escolar mais eu me esforço para fazê-lo. Eu leio para aprender coisas novas. 
Exemplos de itens da escala de motivação extrínseca

Eu vou à escola porque meus pais me obrigam.

Eu me esforço porque eu quero que a professora preste atenção em mim.

\section{Procedimento}

\section{Coleta dos dados}

Somente foram convocados a responderem os instrumentos os alunos que foram autorizados, pelos pais, para participar da pesquisa, após assinatura do Termo de Consentimento Livre e Esclarecido. A aplicação dos instrumentos foi realizada em pequenos grupos, com dez alunos cada, em três encontros para cada grupo. No primeiro encontro foi, primeiramente, aplicada a escala de motivação e, em seguida, o subteste de aritmética do TDE. No segundo encontro foi aplicado o subteste de escrita. No terceiro encontro foi realizado individualmente com cada participante o subteste de leitura. O projeto de pesquisa para realização deste estudo foi submetido ao Comitê de Ética e Pesquisa da Faculdade de Ciências Médicas da Educação Unicamp, sendo aprovado em 26 de março de 2008.

\section{Resultados}

Os resultados obtidos são apresentados em duas etapas. Primeiramente são expostos os dados descritivos e comparativos do desempenho escolar dos estudantes e os referentes à motivação. Em seguida são expostos os resultados do teste de correlação de Spearman para cada um dos anos escolares investigados.

\section{Desempenho escolar}

Os resultados do desempenho escolar, discriminados em função dos testes de leitura, escrita, aritmética e do desempenho geral no TDE encontram-se discriminados a seguir. 
TABELA 1 - MÉDIA, DESVIO PADRÃO E ACERTOS EM RELAÇÃO À LEITURA, ESCRITA, ARITMÉTICA E AO TDE GERAL DOS ESTUDANTES DO $3^{\circ}$ ANO ESCOLAR

\begin{tabular}{lcccc}
\hline & $\begin{array}{c}\text { Leitura } \\
(\mathrm{N}=47)\end{array}$ & $\begin{array}{c}\text { Escrita } \\
(\mathrm{N}=49)\end{array}$ & $\begin{array}{c}\text { Aritmética } \\
(\mathrm{N}=50)\end{array}$ & $\begin{array}{c}\text { TDE Geral } \\
(\mathrm{N}=46)\end{array}$ \\
\hline Média & 62,94 & 19,53 & 13,48 & 96,43 \\
DP* & 7,21 & 5,95 & 2,44 & 12,95 \\
Mínimo de acertos & 26 & 4 & 8 & 40 \\
Máximo de acertos & 70 & 30 & 18 & 113 \\
\hline
\end{tabular}

*desvio padrão

No subteste de leitura os resultados demonstraram que a quantidade de acertos dos participantes do terceiro ano escolar variou de 26 a 70 pontos, ou seja, alguns acertaram a leitura de todas as palavras e não houve ninguém que errasse todas as palavras. No subteste de escrita pode-se verificar que houve estudantes que acertaram a escrita de poucas palavras e que nenhum acertou o subteste na sua totalidade. Em aritmética também se verifica que o máximo de acertos para esta amostra foi de 18 questões e que a média de acertos do grupo foi inferior a $50 \%$. O desempenho obtido no teste como um todo também revelou que nenhum dos participantes acertou o teste na sua totalidade e que alguns tiveram um número de acertos muito baixo (40 acertos).

TABELA 2 - MÉDIA, DESVIO PADRÃO, MÍNIMO E MÁXIMO DE ACERTOS EM RELAÇ̃̃O À LEITURA, ESCRITA, ARITMÉTICA E AO TDE GERAL DOS ESTUDANTES DO $4^{\circ}$ ANO ESCOLAR

\begin{tabular}{lcccc}
\hline & $\begin{array}{c}\text { Leitura } \\
(\mathrm{N}=30)\end{array}$ & $\begin{array}{c}\text { Escrita } \\
(\mathrm{N}=35)\end{array}$ & $\begin{array}{c}\text { Aritmética } \\
(\mathrm{N}=31)\end{array}$ & $\begin{array}{c}\text { TDE Geral } \\
(\mathrm{N}=26)\end{array}$ \\
\hline Média & 64,53 & 22,63 & 21,23 & 109,31 \\
DP & 4,36 & 5,71 & 3,08 & 10,57 \\
Mínimo de acertos & 54 & 12 & 11 & 89 \\
Máximo de acertos & 70 & 33 & 26 & 127 \\
\hline
\end{tabular}

No subteste de leitura os resultados do quarto ano demonstraram que alguns estudantes acertaram a leitura de todas as palavras e não houve ninguém que errasse todas. Também se observa que a quantidade mínima de acertos foi maior do que do terceiro ano. No subteste de escrita os acertos variaram de 12 a 33 pontos, podendo-se afirmar que não houve estudantes que acertaram a escrita de todas as palavras do subteste. Em aritmética o máximo de acertos para esta amostra foi de 26 para um total de 38 questões. O desempenho obtido no teste como um todo também revelou que nenhum dos participantes acertou o 
teste na sua totalidade, mas que a quantidade de acertos deste grupo de alunos esteve acima dos $50 \%$.

TABELA 3 - MÉDIA, DESVIO PADRÃO, MÍNIMO E MÁXIMO DE ACERTOS EM RELAÇÃO À LEITURA, ESCRITA, ARITMÉTICA E AO TDE GERAL DOS ESTUDANTES DO $5^{\circ}$ ANO ESCOLAR

\begin{tabular}{lcccc}
\hline & $\begin{array}{c}\text { Leitura } \\
(\mathrm{N}=34)\end{array}$ & $\begin{array}{c}\text { Escrita } \\
(\mathrm{N}=40)\end{array}$ & $\begin{array}{c}\text { Aritmética } \\
(\mathrm{N}=39)\end{array}$ & $\begin{array}{c}\text { TDE Geral } \\
(\mathrm{N}=31)\end{array}$ \\
\hline Média & 66,97 & 25,38 & 22,54 & 114,74 \\
DP & 2,99 & 4,52 & 3,48 & 7,63 \\
Mínimo de acertos & 59 & 12 & 12 & 99 \\
Máximo de acertos & 70 & 33 & 29 & 129 \\
\hline
\end{tabular}

Os resultados dos estudantes de quinto ano revelaram que em leitura também houve estudantes que acertaram a leitura de todas as palavras e não houve ninguém que errasse todas. Quanto à escrita pode-se verificar que nenhum estudante acertou o teste na sua totalidade e quanto à matemática o máximo de acertos foi de 29 questões num total de 38 .

Para verificar se os estudantes, que cursavam diferentes anos escolares, se diferenciaram no desempenho obtido nos diferentes subtestes foi aplicada a prova estatística não paramétrica de Kruskall-Wallis que revelou serem as diferenças estatisticamente significantes para a leitura $(p=0,001)$, escrita $(p \leq 0,001)$, aritmética $(p \leq 0,001)$ e TDE geral $(p \leq 0,001)$. Em leitura verificou-se que o desempenho dos estudantes de quarto ano foi semelhante ao dos alunos de terceiro, apesar de terem um ano a mais de escolarização. Já os alunos de quinto ano tiveram um desempenho melhor nesta prova quando comparados aos alunos de terceiro e quarto anos. Para a escrita observou-se que, como esperado, o avanço na escolaridade correspondeu a uma melhora na realização da escrita de palavras dos estudantes de diferentes anos escolares. Em aritmética verificou-se que as diferenças estiveram localizadas entre os estudantes do terceiro e quarto anos e entre o terceiro e quintos anos, sendo que os estudantes das séries mais avançadas se saíram melhor na referida prova. Destaca-se, no entanto, que os estudantes de quarto e quinto anos tiveram um desempenho semelhante nesta prova, apesar da diferença de um ano de escolarização.

\section{Motivação}

Os resultados obtidos com a escala de motivação encontram-se na Tabela 4. 
TABELA 4 - RESULTADOS DA MOTIVAÇÃO INTRÍNSECA E EXTRÍNSECA POR ANO ESCOLAR

\begin{tabular}{lcccccc}
\hline & \multicolumn{3}{c}{ Motivação Intrínseca } & \multicolumn{3}{c}{ Motivação Extrínseca } \\
& $3^{\circ}$ ano & $4^{\circ}$ ano & $5^{\circ}$ ano & $3^{\circ}$ ano & $4^{\circ}$ ano & $5^{\circ}$ ano \\
& $\mathrm{N}=43$ & $\mathrm{~N}=34$ & $\mathrm{~N}=40$ & $\mathrm{~N}=43$ & $\mathrm{~N}=34$ & $\mathrm{~N}=40$ \\
\hline Média & 18,65 & 17,24 & 16,85 & 9,95 & 8,68 & 7,13 \\
DP & 1,90 & 2,47 & 2,70 & 5,71 & 4,86 & 4,39 \\
Pontuação Mínima & 11 & 11 & 11 & 0 & 1 & 0 \\
Pontuação Máxima & 20 & 20 & 20 & 20 & 19 & 18 \\
\hline
\end{tabular}

Com o intuito de verificar se existia um predomínio de uma orientação motivacional mais intrínseca ou extrínseca, aplicou-se a prova de Wilcoxon ( $\mathrm{z}=$ $-8,66$ e $\mathrm{p} \leq 0,001$ ), que revelou maior motivação intrínseca (média 17,62) que extrínseca (média 8,62) entre os estudantes desta amostra. O teste de Kruskall-Wallis permitiu, por sua vez, comparar a motivação dos estudantes nos diferentes anos escolares, revelando ser estatisticamente significantes a motivação intrínseca $(\mathrm{p}=0,002)$ e a motivação extrínseca $(\mathrm{p}=0,05)$. Com o teste $U d e$ Mann-Whitney foi possível verificar que, em relação à motivação intrínseca as diferenças estiveram presentes entre os estudantes de terceiro e quarto anos ( $U=$ $465,00$ e $z=-2,80)$ com um valor de probabilidade de 0,005 e, para o terceiro e quinto anos $(U=520,50, z=-3,17)$, com probabilidade de 0,002 . Essas diferenças indicam maior motivação intrínseca nos alunos de $3^{\circ}$ ano em relação aos de $4^{\circ}$ e $5^{\circ}$ anos. Os estudantes de quarto e quinto anos não apresentaram diferenças quanto à motivação intrínseca. Em relação à motivação extrínseca verificou-se que somente os estudantes de terceiro e quinto anos se diferenciaram entre si $(U=605,00, z=-2,32$ e $\mathrm{p}=0,020)$ indicando neste caso que os alunos de terceiro ano revelaram-se mais motivados extrinsecamente que os de quinto ano.

\section{Relação entre desempenho escolar e motivação}

As relações entre o desempenho dos estudantes no TDE e a motivação escolar foram verificadas por meio da análise de correlação de Spearman e revelaram que, para os estudantes de terceiro ano escolar, essas correlações não foram verificadas em nenhum dos conteúdos avaliados. Para os estudantes de quarto ano verificou-se que a motivação intrínseca correlacionou-se positiva- 
mente e de forma moderada com o desempenho em escrita $(\mathrm{r}=0,58, \mathrm{p} \leq 0,001)$ e com o desempenho geral $(\mathrm{r}=0,51, \mathrm{p}=0,01)$ revelando que quanto maior a motivação intrínseca melhor o desempenho no subteste de escrita e no teste como um todo. No que diz respeito à motivação extrínseca também houve uma correlação negativa e baixa apenas com a escrita $(r=-0,35, p=0,04)$ revelando que, quanto menor a motivação extrínseca maior o desempenho dos estudantes na prova de escrita. Para os demais conteúdos não foram verificadas relações.

O resultado referente ao quinto ano escolar revelou correlação positiva e moderada entre o desempenho geral no TDE e a motivação intrínseca $(r=0,48$, $\mathrm{p}=0,006)$ e correlação negativa e baixa com a motivação extrínseca $(\mathrm{r}=-0,36$, $p=0,045)$, mas não entre os conteúdos específicos. Neste caso os estudantes que manifestaram uma maior motivação intrínseca também apresentaram desempenho melhor no TDE e, por outro lado, uma menor motivação extrínseca esteve correlacionada também com um maior desempenho no TDE.

\section{Discussão}

Os estudos realizados nas últimas décadas argumentam que a motivação interfere consideravelmente no desempenho escolar dos alunos por realçar o comportamento de aprendizagem e de realização dos indivíduos, tais como escolher tarefas, prestar atenção, despender esforços e demonstrar persistência. Apesar da importância atribuída a essa variável, os estudos realizados sobre a temática da motivação escolar têm revelado que a sua compreensão no funcionamento individual não é tão simples quanto possa parecer e as relações com o desempenho escolar de estudantes não são conclusivas.

Os achados neste estudo, em relação à motivação dos estudantes, revelam que, como já apontado por outros realizados em diferentes contextos e em diferentes épocas, os alunos se mostram mais motivados, tanto intrínseca quanto extrinsecamente, nos anos iniciais da escolarização e que a motivação tende a decrescer com o avanço da escolarização (LEPPER; CORPUS; IYENGAR, 2005; MARTINELLI, 2008; MARTINELLI; SISTO, 2010; OTIS; GROUZET; PELLETIER, 2005). Outro dado revelado por este estudo é que a motivação intrínseca prevaleceu sobre a extrínseca. Esse resultado é semelhante ao verificado no estudo de Neves e Boruchovitch (2004) e no de Paiva e Boruchovitch (2010).

Os resultados revelados por estes estudos também reforçam aquilo que a literatura tem defendido sobre as relações entre o desempenho escolar e a motivação. No entanto, deve-se destacar que essas relações não foram confirmadas na 
sua totalidade, uma vez que elas não se confirmam para todos os anos escolares analisados e nem tampouco para os conteúdos específicos. Assim, com relação aos conteúdos específicos, correlações entre motivação e desempenho em escrita foram verificadas apenas entre os estudantes de quarto ano. Essas diferenças revelaram que quanto maior a motivação intrínseca melhor o desempenho na escrita e quanto maior motivação extrínseca menor desempenho nesta prova. No presente estudo não foram verificadas relações entre a motivação, intrínseca ou extrínseca, e o desempenho atingido em aritmética. Da mesma maneira, no que se refere à leitura não se verificaram relações significativas entre o desempenho nesta prova e a motivação.

As análises do desempenho geral no TDE, o que incluía as provas de leitura, escrita e aritmética, revelaram apenas uma maior motivação intrínseca por parte dos estudantes do quarto ano, mas nenhuma relação com a motivação extrínseca. Já para os alunos do quinto ano, a relação estabelecida foi ter melhor desempenho no teste associado à maior motivação intrínseca e menor desempenho associado à maior motivação extrínseca. No estudo conduzido por Martinelli e Genari (2009) as autoras também encontraram uma correlação significativa e positiva entre desempenho e motivação intrínseca (apenas para os alunos do quinto ano), e uma correlação significativa e negativa entre desempenho e motivação extrínseca para os alunos do quarto e quintos anos.

$\mathrm{O}$ presente estudo, e os demais analisados, tem indicado de forma mais consistente o fato de a motivação se correlacionar com o desempenho de estudantes de uma forma geral. No entanto, ainda não se tem dados consistentes sobre como a motivação encontra-se relacionada ao desempenho de conteúdos específicos. Considera-se que talvez esta seja uma tarefa mais difícil e a princípio se pontuam duas situações que precisam ser analisadas. Primeiro, o fato de que o instrumento de motivação avalia a percepção da criança de forma geral e não contextualizada, ou seja, não se investigou a motivação para a escrita, leitura e aritmética. Com certeza as respostas poderiam ter sido diferentes se tivesse sido solicitado à criança que respondesse pensando sobre esses conteúdos. Essa análise remete ao segundo ponto de destaque, que é o fato de se saber que somos influenciados, em nossas avaliações, pelas experiências que tivemos ou que estamos vivenciando no momento.

Assim, como já pontuado, essas situações podem ter interferido nos resultados encontrados, e embora não se tenha conseguido demonstrar de forma consistente as relações entre motivação e desempenho em conteúdos específicos, se pode apontar para alguns indícios desta relação, como o verificado na escrita, e reafirmar as relações entre motivação e o desempenho visto de uma forma mais geral. Fazem-se necessárias, também, novas investigações referentes à motivação extrínseca e suas relações com a motivação intrínseca, 
uma vez que alguns autores como Ryan, Connell e Grolnick (1992) criticam a ideia de que a motivação extrínseca seja prejudicial aos comportamentos mais autorregulados. Harter (1981) também afirmou que, em determinadas situações, o interesse intrínseco e a recompensa extrínseca se complementam, ou seja, fatores internos e externos se relacionam para produzir um comportamento intrinsecamente motivado.

Embora neste estudo não se tenha investigado a motivação no sentido de identificar uma possível interiorização dos comportamentos extrinsecamente motivados, e nem como o interesse intrínseco possa se relacionar com o extrínseco, ficou evidente que o desempenho se correlacionou de forma positiva com a orientação motivacional intrínseca e negativa com a extrínseca. Como é evidente que as duas orientações encontram-se presentes entre os estudantes, talvez fosse interessante que as escolas aproveitassem a motivação extrínseca para trabalhar no sentido de destacar a valorização da tarefa e valorizar e reconhecer os esforços feitos pelos alunos. Segundo Ryan, Connell e Grolnick (1992), a valorização seria um caminho para a interiorização da motivação por ativar o sentimento de competência e reforçar o sentimento de autonomia, características presentes na motivação intrínseca.

Sem dúvida alguma, no que se refere ao processo de aprendizagem, há muito o que explorar. Assim, neste estudo foi possível investigar alguns dos fatores que interferem na aprendizagem, especificamente a motivação em estudantes do ensino fundamental. No entanto, considera-se importante que outros estudos possam ampliar a amostra de estudantes com vistas a buscar dados mais abrangentes sobre essa questão, tendo em vista que o presente estudo trabalhou com um número reduzido de participantes e de apenas uma escola. Considera-se também pertinente que se aprofunde cada vez mais, e sob novas perspectivas, os diversos aspectos que, de forma direta ou indireta, podem influenciar o desempenho escolar. Uma melhor compreensão sobre os processos e as variáveis que influenciam o desempenho escolar possibilitará que melhores e mais eficazes intervenções sejam desenvolvidas e implementadas, de modo que na escola se promova, de forma mais efetiva, o sucesso acadêmico.

\section{REFERÊNCIAS}

BZUNECK, J. A. A motivação do aluno: aspectos introdutórios. In: BORUCHOVITCH, E.; BZUNECK, J. A. (Org). A motivação do aluno: contribuições da psicologia contemporânea. Petropólis, RJ: Vozes, 2001. p. 9-36. 
CORPUS, J. M.; MCCLINTIC-GILBERT, M.; HAYENGA, A. O. Within-year changes in children's intrinsic and extrinsic motivational orientations: Contextual predictors and academic outcomes. Contemporary Educational Psychology, v. 34, n. 2, p. 154-166, 2009.

COSTA, G. D. F. Relações entre as orientações motivacionais e o desempenho escolar de alunos da $7^{a}$ Série do Ensino Fundamental em Matemática na resolução de equação do $1^{\circ}$ grau.166f. Dissertação (Mestrado em Educação) - Faculdade de Educação, Universidade Estadual de Campinas, Campinas, SP, 2005.

DECI, E. L.; RYAN, R. M. Intrinsic motivation and self-determination in human behavior. New York: Plenum. 1985. 263 p.

GILLET, N.; VALLERAND, R. J.; LAFRENIÈRE, M. K. Intrinsic and extrinsic school motivation as a function of age: the mediating role of autonomy support. Social Psychology of Education, v. 15, n. 1, p. 77-95, 2012.

GOTTFRIED, A. E. Academic intrinsic motivation in elementary and junior high school students. Journal of Educational Psychology, v. 77, n. 6, p. 631-664, 1985.

GOTTFRIED, A. E. et al. Multivariate latent change modeling of developmental decline in academic intrinsic math motivation and achievement: Childhood through adolescence. International Journal of Behavioral Development, v. 31, n. 4, p. 317-327, 2007.

GUIMARÃES, S. R.; BORUCHOVITCH, E. O estilo motivacional do professor e a motivação intrínseca dos estudantes: uma perspectiva da Teoria da Autodeterminação. Psicologia: Reflexão e Crítica, v. 17, n. 2, p. 143-150, 2004.

HARTER, S. A new self-report scale of intrinsic orientation in the classroom: Motivational and Informational Components. Developmental Psychology, v. 17, n. 3, p. 300-312, 1981.

HIGA, S. L.; MARTINELLI, S. C. As Orientações Motivacionais de Estudantes do Ensino Fundamental. Teoria e Prática da Educação, v. 9, n. 2, p. 169-177, 2006.

LEPPER, M. R.; CORPUS, J. H.; IYENGAR, S. S. Intrinsic and Extrinsic Motivational Orientations in the Classroom: Age Differences and Academic Correlates. Journal of Educational Psychology, v. 97, n. 2, p. 184-196, 2005.

MARTINELLI, S. C. A study on the motivation among elementary school children. Anais do V Congreso Internacional de Psicología y Educación: Los retos del Futuro, Oviedo, Espanha, 2008. 1 CD.

MARTINELLI, S. C.; GENARI, C. H. Relações entre desempenho escolar e orientações motivacionais. Estudos em Psicologia, Natal, v. 14, n. 1, p. 13-21, 2009.

MARTINELLI, S. C.; SISTO, F. F. Escala para avaliação da motivação escolar infanto-juvenil - EAME-IJ. São Paulo: Casa do Psicólogo, 2011, 79 p.

MARTINELLI, S. C.; SISTO, F. F. Motivação de estudantes: um estudo com crianças do ensino fundamental. Revista de Avaliação Psicológica, v. 9, n. 3, p. 413-420, 2010. 
NEVES, E. R. C.; BORUCHOVITCH, E. A motivação dos alunos no contexto da progressão continuada. Psicologia: Teoria e Pesquisa, v. 20, n. 1, p. 44-56, 2004.

OTIS, N.; GROUZET, F. M. E.; PELLETIER, L. G. Latent Motivational Change in an Academic Setting: A 3-Year Longitudinal Study. Journal of Educational Psychology, v. 97, n. 2, p. 170-183, 2005.

PAIVA, M. L. P.; BORUCHOVITCH, E. Orientações motivacionais, crenças educacionais e desempenho escolar de estudantes do Ensino Fundamental. Psicologia em Estudo, v. 15, n. 2, p. 235-244, 2010.

RYAN, R. M.; CONNELL, J. P.; DECI, E. L. A motivational analysis of self-determination and self-regulation in education. In: AMES, C.; AMES, R. (Org.). Research on motivation in education. New York: Academic Press, 1985. p. 16-31.

RYAN, R. M.; CONNELL, J. P.; GROLNICK, W. S. When achievement is not intrinsically motivated: A theory of internalization and self-regulation in school. In: BOGGIANO, A.; PITTMAN, T. (Ed). Achievement motivation: a social-developmental perspective. Cambridge: Cambridge University Press, 1992. p. 167-188.

RYAN, R. M.; DECI, E. L. Self-Determination Theory and the Facilitation of Intrinsic Motivation, Social Development and Well-Being. American Psychologist, v. 55, n. 1, p. 68-78, 2000.

SÁ, I. Os componentes motivacionais da aprendizagem auto-regulada. In: SILVA, A.; DUARTE, A. M.; SÁ, I.; SIMÃO, A. M. V. (Org.). Aprendizagem Auto-regulada pelo Estudante: perspectivas psicológicas e educacionais. Porto: Porto Editora, 2004. p. 55-75.

STEIN, L. M. TDE: Teste de Desempenho Escolar: manual para a aplicação e interpretação. São Paulo: Casa do Psicólogo, 1994.

Texto recebido em 27 de maio de 2012. Texto aprovado em 06 de maio de 2014. 